\author{
Applicable Analysis and Discrete Mathematics \\ available online at http://pefmath.etf.rs
}

Appl. Anal. Discrete Math. 9 (2015), 347-356.

doi:10.2298/AADM150908017C

\title{
SUPERCONNECTIVITY OF NETWORKS MODELED BY THE STRONG PRODUCT OF GRAPHS
}

\author{
R. M. Casablanca, M. Cera, P. García-Vázquez \\ J. C. Valenzuela-Tripodoro
}

\begin{abstract}
Maximal connectivity and superconnectivity in a network are two important features of its reliability. In this paper, using graph terminology, we first give a lower bound for the vertex connectivity of the strong product of two networks and then we prove that the resulting structure is more reliable than its generators. Namely, sufficient conditions for a strong product of two networks to be maximally connected and superconnected are given.
\end{abstract}

\section{INTRODUCTION}

In a multiprocessor system, processors communicate by exchanging messages through an interconnection network whose topology is often modeled by an undirected graph $G=(V, E)$, where every node in $V$ corresponds to a processor, and every edge in $E$ corresponds to a communication link. The properties of the graph determine the systems working efficiency. When selecting or designing an interconnection network, many of mutually conflicting requirements correspond to measures in a graph as density, size, average degree, diameter, connectivity, etc. Since it is almost impossible to design an optimal network for all conditions, the selection criterion must be determined in advance. One of the most desirable criterions for the design of a large interconnection network joins together the requirements of high reliability and small maximum transmission delay between nodes of the network. So, making use of Graph Theory, the primary aim is to get a strong connectivity joint to a suitable diameter in certain large graphs.

\footnotetext{
2010 Mathematics Subject Classification. 05C40.

Keywords and Phrases. Networks reliability, connectivity, strong product graphs, maximally connected graphs, superconnected graphs.
} 
It is well known that the product of graphs is an important research topic in Graph Theory (see, e.g. $[\mathbf{3}, \mathbf{2 1}, \mathbf{2 4}]$ ). This graph operation has been extensively studied in a wide range of subjects, including connectivity $[\mathbf{5}, \mathbf{2 4}]$, geodetic $[\mathbf{6}]$, bandwidth [18] and roman domination [9], among others. A fundamental principle for network design is extendability. That is to say, the possibility of building larger versions of a network preserving certain desirable properties. For designing largescale interconnection networks, the strong product is a useful method for obtaining large graphs from smaller ones whose invariants can be easily calculated.

The problem of computing graph products was applied several years ago in a theoretical biology context [25]. The authors provided a concept concerning the topological theory of the relationships between genotypes and phenotypes. A phenotype space inherits its structure from an underlying sequence space. The structure of localized subsets turns out to be of particular interest. GavriLETS and Gravner [8], Grüner et al. [10], and Reidys and Stadler [22], for example, describe subgraphs in sequence spaces that correspond to the subset of viable genomes or to those sequences that give rise to the same phenotype. The structure of these subgraphs is intimately related to the dynamics of evolutionary processes $[20]$.

Other applications of graph products can be found in rather different areas such as computer graphics and theoretical computer science. In $[\mathbf{1}, \mathbf{2}]$, the authors provide a framework, called TopoLayout, to draw undirected graphs based on the topological features they contain. Topological features are detected recursively, and their subgraphs are collapsed into single nodes, forming a graph hierarchy. Graph products have a well understood structure, that can be drawn in an effective way. Hence, for an extension of this framework approximate graph products are of a particular interest. Reasons and motivations to study graph products or graphs that have a product-like structure can be found in many other areas, e.g. for the formation of finite element models or construction of localized self-equilibrating systems in computational engineering $[\mathbf{1 5}, \mathbf{1 6}, \mathbf{1 7}]$. Other motivations can be found in discrete mathematics. A natural question is what can be said about a graph invariant of a graphs product if one knows the corresponding invariants of the factors. There are many contributions treating this problem, e.g. $[\mathbf{5}, \mathbf{1 1}, \mathbf{1 3}$, $19,24]$.

In all applications of practical interest, the graphs product in question needs to be analyzed in a way that is robust against inaccuracies, noise, and perturbations in the data. However, these results have to be either obtained from computer simulations or they need to be estimated from measured data. In both cases, they are known only approximately. In order to deal with such inaccuracies, exact solution based on theoretical mathematical reasoning needs to be found.

The network robustness should be analyzed from different perspectives. In this paper we analyze the connectivity parameter $\kappa$, which represents the minimum number of nodes that must fail to disrupt the communication between at least one pair of nodes in the network (see [14]). In [24] ŠPACAPAN gives the following lower bound for the connectivity of the strong product $G_{1} \otimes G_{2}$ of two connected 
networks, modeled by two graphs $G_{1}$ and $G_{2}$ :

$$
\kappa\left(G_{1} \otimes G_{2}\right) \geq \min \left\{\kappa\left(G_{1}\right)\left(1+\delta\left(G_{2}\right)\right), \kappa\left(G_{2}\right)\left(1+\delta\left(G_{1}\right)\right)\right\} .
$$

We obtain an improvement of this bound and moreover, we prove sufficient conditions on the girth and the minimum degree of two connected networks to be superconnected. These conditions show that the strong product is a useful method to extend a given network to a larger and much more reliable one so that the maximum communication delay between two nodes of the new network is approximately the same as that of the original one.

\section{GRAPH THEORETICAL PRELIMINARIES}

Throughout this paper, all the graphs are simple, that is, with neither loops nor multiple edges. Notations and terminology not explicitly given here can be found in the book by CHARTRAND and LESNIAK [7].

Let $G$ be a graph with vertex set $V=V(G)$ and edge set $E=E(G)$. The cardinalities of these sets are denoted by $|V(G)|$ and $|E(G)|$. Let $u$ and $v$ be two distinct vertices of $G$. A path from $u$ to $v$, also called an $u v$-path in $G$, is a subgraph $P$ with vertex set $V(P)=\left\{u=x_{0}, x_{1}, \ldots, x_{r}=v\right\}$ and edge set $E(P)=\left\{x_{0} x_{1}, \ldots, x_{r-1} x_{r}\right\}$. This path is usually denoted by $P: x_{0} x_{1} \cdots x_{r}$ and $r$ is the length of $P$. A cycle in $G$ of length $r$ is a path $\mathcal{C}_{r}: x_{0} x_{1} \cdots x_{r}$ such that $x_{0}=x_{r}$. The girth of $G$, denoted by $g(G)$, is the length of a shortest cycle in $G$, if any. Otherwise, we set $g(G)=\infty$. The set of adjacent vertices to $v \in V(G)$ is denoted by $N_{G}(v)$. The degree of $v$ is $d_{G}(v)=\left|N_{G}(v)\right|$, whereas $\delta(G)=\min _{v \in V(G)} d_{G}(v)$ and $\Delta(G)=\max _{v \in V(G)} d_{G}(v)$ stand for the minimum degree and the maximum degree of $G$, respectively. A complete bipartite graph $K_{m, n}$ is a graph whose vertices can be partitioned into two subsets $V_{1}$ and $V_{2}$, with cardinalities $m$ and $n$, respectively, such that no edge has both endpoints in the same subset and each vertex in $V_{1}$ is adjacent to every vertex of $V_{2}$.

The diameter of $G$ is written as $D(G)$, which is finite if $G$ is connected. A graph is said to be connected if for every pair of vertices there is a path connecting them. A cut set of a connected graph $G$ is a set $S$ of vertices such that $G-S$ is not connected or is an isolated vertex. Each connected subgraph of $G-S$ is called a component of $G-S$. The (vertex)-connectivity of $G$, denoted by $\kappa(G)$, is the minimum cardinality of a cut set, and it is widely known that $\kappa(G) \leq \delta(G)$. A connected graph $G$ is called maximally connected if $\kappa(G)=\delta(G)$. A connected graph $G$ is superconnected if for every minimum cut set $S$ in $G$, the graph $G-S$ has an isolated vertex. Observe that every superconnected graph is maximally connected but the converse is not true. It is easy to see from the cycle graph $\mathcal{C}_{n}$, $n \geq 6$.

The construction of new graphs from two given ones is not complicated. Basically, the method consists of joining together several copies of one graph according to the structure of another one, the latter being usually called the main graph of 
the construction. Since 1960 some relevant graph theory researchers have defined different types of graph products. The main difference between them comes from the number of intercopy edges and the connection criterion. One of these products of graphs is the strong product of two given graphs, and it was defined in [23] by Sabidussi in the following way.

Definition $1([\mathbf{2 3}])$. Let $G_{1}=\left(V\left(G_{1}\right), E\left(G_{1}\right)\right)$ and $G_{2}=\left(V\left(G_{2}\right), E\left(G_{2}\right)\right)$ be two graphs. The strong product $G_{1} \otimes G_{2}$ of $G_{1}$ and $G_{2}$ has $V\left(G_{1}\right) \times V\left(G_{2}\right)$ as vertex set, so that two distinct vertices $\left(x_{1}, x_{2}\right)$ and $\left(y_{1}, y_{2}\right)$ of $G_{1} \otimes G_{2}$ are adjacent if $x_{1}=y_{1}$ and $x_{2} y_{2} \in E\left(G_{2}\right)$, or $x_{1} y_{1} \in E\left(G_{1}\right)$ and $x_{2}=y_{2}$, or $x_{1} y_{1} \in E\left(G_{1}\right)$ and $x_{2} y_{2} \in E\left(G_{2}\right)$.

From the definition, it clearly follows that the strong product of two graphs is commutative. Indeed, $G_{1} \otimes G_{2}$ can be seen as the graph formed by $\left|V\left(G_{1}\right)\right|$ copies of $G_{2}$, $G_{2}^{x_{1}}, \ldots, G_{2}^{x_{n}}$, corresponding to the set of vertices $V\left(G_{1}\right)=\left\{x_{1}, \ldots, x_{n}\right\}$, and moreover, for every edge $x_{i} x_{j} \in E\left(G_{1}\right)$ and every vertex $y \in V\left(G_{2}\right)$, vertex $\left(x_{i}, y\right)$ is adjacent in $G_{1} \otimes G_{2}$ to each vertex of $\bigcup_{z \in N_{G_{2}}[y]}\left(x_{j}, z\right)$. This latter way of construction of $G_{1} \otimes G_{2}$ can be expressed by exchanging $G_{1}$ and $G_{2}$ (see, for instance, Figure 1).

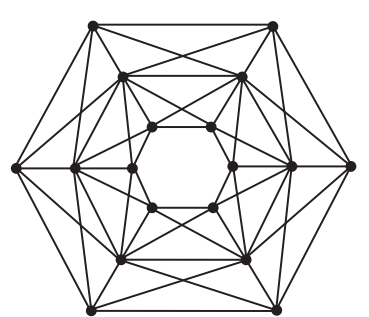

Figure 1. The strong product of a cycle of length 6 and a path of length 2 .

Some properties regarding the minimum degree, the maximum degree and the diameter of $G_{1} \otimes G_{2}$ can be found in [12].

Lemma $2([\mathbf{1 2}])$. Let $G_{1}$ and $G_{2}$ be two graphs. Then:

(i) $\delta\left(G_{1} \otimes G_{2}\right)=\delta\left(G_{1}\right) \delta\left(G_{2}\right)+\delta\left(G_{1}\right)+\delta\left(G_{2}\right)$.

(ii) $\Delta\left(G_{1} \otimes G_{2}\right)=\Delta\left(G_{1}\right) \Delta\left(G_{2}\right)+\Delta\left(G_{1}\right)+\Delta\left(G_{2}\right)$.

(iii) If both $G_{1}$ and $G_{2}$ are connected, then $G_{1} \otimes G_{2}$ is also connected and

$$
D\left(G_{1} \otimes G_{2}\right)=\max \left\{D\left(G_{1}\right), D\left(G_{2}\right)\right\} .
$$

\section{MAIN RESULTS}

Let $S_{1}$ and $S_{2}$ be cut sets of $G_{1}$ and $G_{2}$, respectively. Then $S_{1} \times V\left(G_{2}\right)$ and $V\left(G_{1}\right) \times S_{2}$ are called an $I$-set of $G_{1} \otimes G_{2}$. Let us denote by $A_{1}, \ldots, A_{k}$ and $B_{1}, \ldots, B_{\ell}$ the components of $G_{1}-S_{1}$ and $G_{2}-S_{2}$, respectively. Then for every $i \in\{1, \ldots, k\}$ and every $j \in\{1, \ldots, \ell\}$, the set $\left(S_{1} \times V\left(B_{j}\right)\right) \cup\left(S_{1} \times S_{2}\right) \cup\left(V\left(A_{i}\right) \times S_{2}\right)$ is called an $L$-set of $G_{1} \otimes G_{2}$. These sets of vertices were introduced in [24] and the following theorem was proved.

Theorem 3 ([24]). Let $G_{1}$ and $G_{2}$ be two connected graphs. Then every cut set in $G_{1} \otimes G_{2}$ of minimum cardinality is either an I-set or an L-set in $G_{1} \otimes G_{2}$. 
The cardinality of an $I$-set in $G_{1} \otimes G_{2}$ can be easily lower bounded. Indeed, if $S$ is a cut set of $G_{1} \otimes G_{2}$ of minimum cardinality and $S$ is an $I$-set, then $\kappa\left(G_{1} \otimes G_{2}\right)=|S|=\min \left\{\kappa\left(G_{1}\right)\left|V\left(G_{2}\right)\right|,\left|V\left(G_{1}\right)\right| \kappa\left(G_{2}\right)\right\}$. Nevertheless, this does not happen when $S$ is an $L$-set.

First we obtain a lower bound of the index of connectivity of the strong product $G_{1} \otimes G_{2}$ of two connected graphs $G_{1}$ and $G_{2}$.

Theorem 4. Let $G_{1}$ and $G_{2}$ be two connected graphs and $G=G_{1} \otimes G_{2}$. If $g\left(G_{1}\right) \geq 4$ then

$$
\min \left\{\left|V\left(G_{1}\right)\right| \kappa\left(G_{2}\right), \kappa\left(G_{1}\right)\left|V\left(G_{2}\right)\right|, \delta\left(G_{1}\right) \kappa\left(G_{2}\right)+\delta\left(G_{1}\right)+\kappa\left(G_{2}\right)\right\} \leq \kappa(G) \leq \delta(G) .
$$

Proof. Clearly, $\kappa(G) \leq \delta(G)$ holds, so we must only prove the other inequality. Denote by

$$
M\left(G_{1}, G_{2}\right)=\min \left\{\left|V\left(G_{1}\right)\right| \kappa\left(G_{2}\right), \kappa\left(G_{1}\right)\left|V\left(G_{2}\right)\right|, \delta\left(G_{1}\right) \kappa\left(G_{2}\right)+\delta\left(G_{1}\right)+\kappa\left(G_{2}\right)\right\} .
$$

Let $S \subset V(G)$ be a cut set of $G$ with $|S|=\kappa(G)$. From Theorem 3 it follows that $S$ is either an $I$-set or an $L$-set. If $S$ is an $I$-set then

$$
|S|=\min \left\{\left|V\left(G_{1}\right)\right| \kappa\left(G_{2}\right), \kappa\left(G_{1}\right)\left|V\left(G_{2}\right)\right|\right\} \geq M\left(G_{1}, G_{2}\right) .
$$

Then suppose that $S=\left(S_{1} \times V\left(B_{j}\right)\right) \cup\left(S_{1} \times S_{2}\right) \cup\left(V\left(A_{i}\right) \times S_{2}\right)$ is an $L$-set, for some $i \in\{1, \ldots, k\}$ and $j \in\{1, \ldots, \ell\}$, being $A_{1}, \ldots, A_{k}$ and $B_{1}, \ldots, B_{\ell}$ the components of $G_{1}-S_{1}$ and $G_{2}-S_{2}$, respectively. If $\left|S_{1}\right| \geq \delta\left(G_{1}\right)$ then $|S| \geq$ $\left|S_{1}\right|+\left|S_{1}\right|\left|S_{2}\right|+\left|S_{2}\right| \geq \delta\left(G_{1}\right)+\delta\left(G_{1}\right) \kappa\left(G_{2}\right)+\kappa\left(G_{2}\right) \geq M\left(G_{1}, G_{2}\right)$ and we are done. Thus, assume that $\left|S_{1}\right|<\delta\left(G_{1}\right)$. In this case, there are at least two adjacent vertices $u, v \in V\left(A_{i}\right)$, since $G_{1}$ is connected. As $G_{1}$ has girth at least 4 , we have $N_{G_{1}}(u) \cap N_{G_{1}}(v)=\emptyset$. Moreover, $N_{G_{1}}(u) \cup N_{G_{1}}(v) \subseteq V\left(A_{i}\right) \cup S_{1}$. Hence, $\left|V\left(A_{i}\right) \cup S_{1}\right| \geq d_{G_{1}}(u)+d_{G_{1}}(v) \geq 2 \delta\left(G_{1}\right)$ and therefore,

$$
\begin{aligned}
|S| & =\left|\left(S_{1} \times V\left(B_{j}\right)\right) \cup\left(S_{1} \times S_{2}\right) \cup\left(V\left(A_{i}\right) \times S_{2}\right)\right| \\
& =\left|\left(S_{1} \times V\left(B_{j}\right)\right) \cup\left(\left(S_{1} \cup V\left(A_{i}\right)\right) \times S_{2}\right)\right| \\
& \geq 1+\left|S_{1} \cup V\left(A_{i}\right)\right|\left|S_{2}\right| \geq 1+2 \delta\left(G_{1}\right) \kappa\left(G_{2}\right) \\
& \geq \delta\left(G_{1}\right) \kappa\left(G_{2}\right)+\delta\left(G_{1}\right)+\kappa\left(G_{2}\right) \geq M\left(G_{1}, G_{2}\right),
\end{aligned}
$$

which completes the proof.

From Theorem 4 and the commutativity of the strong product of two graphs, it follows this theorem whose proof is straightforward.

Theorem 5. Let $G_{1}$ and $G_{2}$ be two connected graphs of order $n_{i}$, minimum degree $\delta_{i}$, connectivity $\kappa_{i}, i=1,2$, and girth at least 4 . Then

$$
\begin{aligned}
& \min \left\{n_{1} \kappa_{2}, \kappa_{1} n_{2}, \max \left\{\delta_{1} \kappa_{2}+\delta_{1}+\kappa_{2}, \kappa_{1} \delta_{2}+\kappa_{1}+\delta_{2}\right\}\right\} \\
& \quad \leq \kappa\left(G_{1} \otimes G_{2}\right) \leq \delta\left(G_{1} \otimes G_{2}\right) .
\end{aligned}
$$


Next we establish sufficient conditions for the strong product of two maximally connected graphs, $G_{1}$ and $G_{2}$, to be maximally connected. These conditions are addressed in terms of the minimum degree and the girth of both $G_{1}$ and $G_{2}$.

To do that we use the well-known Moore bound (see [4] p. 105) which says that every graph with girth $g \geq 3$ and minimum degree $\delta \geq 2$ has at least $n_{0}(\delta, g)$ vertices, where

$$
n_{0}(\delta, g)= \begin{cases}1+\delta \sum_{i=0}^{(g-3) / 2}(\delta-1)^{i}, & \text { if } g \text { is odd } \\ 2 \sum_{i=0}^{g / 2-1}(\delta-1)^{i}, & \text { if } g \text { is even. }\end{cases}
$$

Theorem 6. Let $G_{1}$ and $G_{2}$ be two connected graphs with at least 3 vertices and girth at least 4 . Then $G_{1} \otimes G_{2}$ is maximally connected if both $G_{1}$ and $G_{2}$ are maximally connected and one of the following assertions holds:

(i) One graph has minimum degree 1 and the other graph has girth at least 5 .

(ii) $\delta\left(G_{i}\right) \geq 2, i=1,2$.

Proof. (i) If $\delta\left(G_{1}\right)=1$ and $\delta\left(G_{2}\right)=1$, then $\delta\left(G_{1} \otimes G_{2}\right)=3$. Thus, $\left|V\left(G_{1}\right)\right| \delta\left(G_{2}\right) \geq 3=\delta\left(G_{1} \otimes G_{2}\right)$ and, analogously, $\delta\left(G_{1}\right)\left|V\left(G_{2}\right)\right| \geq 3=\delta\left(G_{1} \otimes G_{2}\right)$. Hence, by Theorem 4 we have

$$
\kappa\left(G_{1} \otimes G_{2}\right)=3=\delta\left(G_{1}\right) \delta\left(G_{2}\right)+\delta\left(G_{1}\right)+\delta\left(G_{2}\right) .
$$

If $\delta\left(G_{1}\right)=1$ and $\delta\left(G_{2}\right) \geq 2$ (the proof is analogous if $\delta\left(G_{1}\right) \geq 2$ and $\delta\left(G_{2}\right)=1$ ), since by hypothesis, $g\left(G_{2}\right) \geq 5$, from the Moore bound (1) it follows that $\left|V\left(G_{2}\right)\right| \geq 1+\delta\left(G_{2}\right)^{2}$. Thus,

$$
\begin{aligned}
\left|V\left(G_{1}\right)\right| \delta\left(G_{2}\right) & \geq \delta\left(G_{1}\right) \delta\left(G_{2}\right)+\delta\left(G_{1}\right)+\delta\left(G_{2}\right)+\left(\left|V\left(G_{1}\right)\right|-3\right) \delta\left(G_{2}\right) \\
& \geq \delta\left(G_{1}\right) \delta\left(G_{2}\right)+\delta\left(G_{1}\right)+\delta\left(G_{2}\right)
\end{aligned}
$$

and

$$
\begin{aligned}
\delta\left(G_{1}\right)\left|V\left(G_{2}\right)\right|=\left|V\left(G_{2}\right)\right| & \geq 1+\delta\left(G_{2}\right)^{2}=\delta\left(G_{1}\right) \delta\left(G_{2}\right)+\delta\left(G_{1}\right)+\delta\left(G_{2}\right)\left(\delta\left(G_{2}\right)-1\right) \\
& \geq \delta\left(G_{1}\right) \delta\left(G_{2}\right)+\delta\left(G_{1}\right)+\delta\left(G_{2}\right) .
\end{aligned}
$$

Therefore, by Theorem 4 we deduce that

$$
\kappa\left(G_{1} \otimes G_{2}\right)=\delta\left(G_{1}\right) \delta\left(G_{2}\right)+\delta\left(G_{1}\right)+\delta\left(G_{2}\right) .
$$

(ii) If $\delta\left(G_{1}\right) \geq 2$ and $\delta\left(G_{2}\right) \geq 2$, then $\left|V\left(G_{i}\right)\right| \geq 2 \delta\left(G_{i}\right), i=1,2$, due to the Moore Bound (1). Using that $a b \geq a+b$ for all $a \geq 2, b \geq 2$, we have

$$
\begin{aligned}
\left|V\left(G_{1}\right)\right| \delta\left(G_{2}\right) & \geq 2 \delta\left(G_{1}\right) \delta\left(G_{2}\right)=\delta\left(G_{1}\right) \delta\left(G_{2}\right)+\delta\left(G_{1}\right) \delta\left(G_{2}\right) \\
& \geq \delta\left(G_{1}\right) \delta\left(G_{2}\right)+\delta\left(G_{1}\right)+\delta\left(G_{2}\right) .
\end{aligned}
$$

Analogously, $\delta\left(G_{1}\right)\left|V\left(G_{2}\right)\right| \geq \delta\left(G_{1}\right) \delta\left(G_{2}\right)+\delta\left(G_{1}\right)+\delta\left(G_{2}\right)$. Hence, $\kappa\left(G_{1} \otimes G_{2}\right)=$ $\delta\left(G_{1}\right) \delta\left(G_{2}\right)+\delta\left(G_{1}\right)+\delta\left(G_{2}\right)$, and the result follows. 
Theorem 6 is best possible in the sense that the hypothesis cannot be relaxed. Indeed, observe, for instance, what happens in the strong product of any cycle $\mathcal{C}_{g}$ of length $g \geq 4$ and the complete graph $K_{n}, n \geq 2$. We can disconnect $\mathcal{C}_{g} \otimes K_{n}$ by removing two copies of $K_{n}$ corresponding to two nonadjacent vertices of $\mathcal{C}_{g}$. Hence, $\kappa\left(\mathcal{C}_{g} \otimes K_{n}\right) \leq 2 n<2(n-1)+n-1+2=\delta\left(\mathcal{C}_{g} \otimes K_{n}\right)$. Analogously, we check that the hypothesis of points $(i)$ and $(i i)$ of Theorem 6 also cannot be relaxed. It suffices to consider the strong product $\mathcal{P}_{r} \otimes \mathcal{C}_{g}$ of a path of length $r \geq 2$ and a cycle of length $g \leq 4$. In this case, by removing one copy of $\mathcal{C}_{g}$ corresponding to any vertex of degree 2 in $\mathcal{P}_{r}$, the resulting graph is disconnected. Thus, $\kappa\left(\mathcal{P}_{r} \otimes \mathcal{C}_{g}\right) \leq g \leq 4<5=\delta\left(\mathcal{P}_{r} \otimes \mathcal{C}_{g}\right)$.

The following lemma will be the key to improve Theorem 6 in the sense that the strong product of two non necessarily maximally connected graphs may be maximally connected or even superconnected.

Lemma 7. Let $G_{1}$ and $G_{2}$ be two graphs of minimum degree at least 2 and let $\delta=\min \left\{\delta\left(G_{1}\right), \delta\left(G_{2}\right)\right\}$. If $\left|V\left(G_{1}\right)\right| \geq 2 \delta\left(G_{1}\right)$ and $\kappa\left(G_{2}\right) \geq \delta\left(G_{2}\right)-\lfloor\delta / 2\rfloor+1$ then $\left|V\left(G_{1}\right)\right| \kappa\left(G_{2}\right) \geq \delta\left(G_{1} \otimes G_{2}\right)$ with equality holds if and only if $\delta$ is even, $\delta\left(G_{i}\right)=\delta$, $i=1,2,\left|V\left(G_{1}\right)\right|=2 \delta$ and $\kappa\left(G_{2}\right)=\delta / 2+1$.

Proof. Denote by $G=G_{1} \otimes G_{2}$. Then

$$
\begin{aligned}
\left|V\left(G_{1}\right)\right| \kappa\left(G_{2}\right) & \geq 2 \delta\left(G_{1}\right)\left(\delta\left(G_{2}\right)-\lfloor\delta / 2\rfloor+1\right) \geq \delta\left(G_{1}\right)\left(2 \delta\left(G_{2}\right)-\delta+2\right) \\
& =\delta(G)+\delta\left(G_{1}\right)\left(\delta\left(G_{2}\right)-\delta+1\right)-\delta\left(G_{2}\right) \\
& \geq \delta(G)+\delta\left(G_{1}\right)+\delta\left(G_{2}\right)-\delta-\delta\left(G_{2}\right) \\
& =\delta(G)+\delta\left(G_{1}\right)-\delta \geq \delta(G) .
\end{aligned}
$$

Hence, $\left|V\left(G_{1}\right)\right| \kappa\left(G_{2}\right) \geq \delta(G)$ where equality holds iff all the previous inequalities become equalities, that is, $\left|V\left(G_{1}\right)\right|=2 \delta\left(G_{1}\right),\lfloor\delta / 2\rfloor=\delta / 2, \delta\left(G_{2}\right)-\delta+1=1$ and $\delta\left(G_{1}\right)-\delta=0$, which completes the proof.

As a consequence of Lemma 7 and making use of the Moore bound (1), we prove that it is possible to construct a superconnected graph by the strong product of two non necessarily maximally connected factors.

Theorem 8. Let $G_{1}$ and $G_{2}$ be two graphs of minimum degree at least 2 and girth at least 4. Let $\delta=\min \left\{\delta\left(G_{1}\right), \delta\left(G_{2}\right)\right\}$. If $\kappa\left(G_{i}\right) \geq \delta\left(G_{i}\right)-\lfloor\delta / 2\rfloor+1, i=1,2$, then $G_{1} \otimes G_{2}$ is maximally connected. Furthermore, $G_{1} \otimes G_{2}$ is superconnected unless $\delta$ is even, $K_{\delta, \delta}$ is one factor and the other factor has minimum degree $\delta$ and connectivity exactly $\delta / 2+1$.

Proof. Denote by $G=G_{1} \otimes G_{2}$. Let $S \subset V(G)$ be a minimum cut set of $G$. By applying Theorem 3, the set $S$ must be either an $I$-set or an $L$-set. If $S$ is an $I$-set then we have $|S|=\min \left\{\left|V\left(G_{1}\right)\right| \kappa\left(G_{2}\right), \kappa\left(G_{1}\right)\left|V\left(G_{2}\right)\right|\right\}$. Without loss of generality we may suppose that $\left|V\left(G_{1}\right)\right| \kappa\left(G_{2}\right)=\min \left\{\left|V\left(G_{1}\right)\right| \kappa\left(G_{2}\right), \kappa\left(G_{1}\right)\left|V\left(G_{2}\right)\right|\right\}$, due to the commutativity of the strong product of two graphs. As $g\left(G_{1}\right) \geq 4$, by the Moore bound (1), we have $\left|V\left(G_{1}\right)\right| \geq 2 \delta\left(G_{1}\right)$. Then both $G_{1}$ and $G_{2}$ satisfy the 
hypothesis of Lemma 7 and therefore, $\left|V\left(G_{1}\right)\right| \kappa\left(G_{2}\right) \geq \delta(G)$ with equality holds iff $\delta$ is even, $\delta\left(G_{i}\right)=\delta, i=1,2,\left|V\left(G_{1}\right)\right|=2 \delta$ and $\kappa\left(G_{2}\right)=\delta / 2+1$. Using the notation of the Moore bound (1), we have $\left|V\left(G_{1}\right)\right|=n_{0}(\delta, 4)$ and it is well known that the only graph of order $n_{0}(\delta, 4)$ and girth at least 4 is the complete bipartite $K_{\delta, \delta}$. Hence, the $I$-set $S$ is a minimum cut set of $G$ iff $\delta$ is even, $G_{1}=K_{\delta, \delta}$, $\delta\left(G_{2}\right)=\delta$ and $\kappa\left(G_{2}\right)=\delta / 2+1$.

Now suppose that $S=\left(S_{1} \times V\left(B_{j}\right)\right) \cup\left(S_{1} \times S_{2}\right) \cup\left(V\left(A_{i}\right) \times S_{2}\right)$ is an $L$-set, for some $i \in\{1, \ldots, k\}$ and $j \in\{1, \ldots, \ell\}$, being $A_{1}, \ldots, A_{k}$ and $B_{1}, \ldots, B_{\ell}$ the components of $G_{1}-S_{1}$ and $G_{2}-S_{2}$, respectively. Reasoning as in the proof of Theorem 4, if $\left|S_{1}\right|<\delta\left(G_{1}\right)$ then $\left|V\left(A_{i}\right) \cup S_{1}\right| \geq 2 \delta\left(G_{1}\right)$ and if $\left|S_{2}\right|<\delta\left(G_{2}\right)$ then $\left|V\left(B_{j}\right) \cup S_{2}\right| \geq 2 \delta\left(G_{2}\right)$. If $\left|S_{1}\right|<\delta\left(G_{1}\right)$ then

$$
\begin{aligned}
|S| & =\left|\left(S_{1} \times V\left(B_{j}\right)\right) \cup\left(\left(S_{1} \cup V\left(A_{i}\right)\right) \times S_{2}\right)\right| \\
& \geq 1+2 \delta\left(G_{1}\right) \kappa\left(G_{2}\right) \geq 1+2 \delta\left(G_{1}\right)\left(\delta\left(G_{2}\right)-\delta / 2+1\right) \\
& =\delta(G)+\delta\left(G_{1}\right)\left(\delta\left(G_{2}\right)-\delta+1\right)-\delta\left(G_{2}\right)+1 \\
& \geq \delta(G)+\delta\left(G_{1}\right)+\delta\left(G_{2}\right)-\delta-\delta\left(G_{2}\right)+1>\delta(G),
\end{aligned}
$$

which contradicts that $S$ is a minimum cut set of $G$. Analogously, we get a contradiction if $\left|S_{2}\right|<\delta\left(G_{2}\right)$. Thus, $\left|S_{i}\right| \geq \delta\left(G_{i}\right)$ for $i=1,2$. Observe that $A_{i} \otimes B_{j}$ is a component of $G-S$. Our aim is to prove that $\left|V\left(A_{i}\right)\right|=\left|V\left(B_{i}\right)\right|=1$ and therefore, $A_{i} \otimes B_{j}$ is an isolated vertex. Otherwise, $|S| \geq\left|S_{1}\right|\left|V\left(B_{j}\right)\right|+\left|S_{1}\right|\left|S_{2}\right|+\left|S_{2}\right|\left|V\left(A_{i}\right)\right|>$ $\left|S_{1}\right|+\left|S_{1}\right|\left|S_{2}\right|+\left|S_{2}\right|=\delta\left(G_{1}\right)+\delta\left(G_{1}\right) \delta\left(G_{2}\right)+\delta\left(G_{2}\right)=\delta(G)$, which is a contradiction. Thus, if the $L$-set $S$ is a minimum cut set then $A_{i} \otimes B_{j}$ is a trivial component of $G-S$, yielding that $G$ is superconnected. This completes the proof.

Theorem 8 is best possible in the sense that the hypothesis cannot be relaxed. First of all, the minimum degree of each factor graph at least 2 must be assumed. Otherwise, the strong product $\mathcal{P}_{r} \otimes \mathcal{C}_{5}$ of a path of length $r \geq 2$ and a cycle of length 5 is a counterexample. In this case, both $\mathcal{P}_{r}$ and $\mathcal{C}_{5}$ are maximally connected graphs and $\kappa\left(\mathcal{P}_{r} \otimes \mathcal{C}_{5}\right)=5=\delta\left(\mathcal{P}_{r} \otimes \mathcal{C}_{5}\right)$. However, the deletion of one copy of $\mathcal{C}_{5}$ corresponding to any vertex of degree 2 in $\mathcal{P}_{r}$, produces a disconnected graph and no component of this graph is an isolated vertex. Therefore, $\mathcal{P}_{r} \otimes \mathcal{C}_{5}$ is not superconnected. In addition, the hypothesis on the connectivity of the factors also must be assumed. If not, observe what happens if $G_{1}$ is formed by two copies of $K_{3,3}$ which share one edge and $G_{2}=K_{3,3}$. Obviously, $\kappa\left(G_{1}\right)=2$ because we can break $G_{1}$ by removing the vertices of the common edge which share the two copies of $K_{3,3}$. Therefore, if we remove in $G_{1} \otimes G_{2}$ the copies of $G_{2}$ corresponding to the minimum cut set of $G_{1}$, we disconnect $G_{1} \otimes G_{2}$ and therefore, $\kappa\left(G_{1} \otimes G_{2}\right) \leq 2\left|V\left(G_{2}\right)\right|=12$. However, $\delta\left(G_{1} \otimes G_{2}\right)=15$, which means that $G_{1} \otimes G_{2}$ is not maximally connected.

Acknowledgements. The authors gratefully acknowledge the helpful comments from the referees. This research was supported by the Ministry of Economy and Competitiveness, Spain, and the European Regional Development Fund (ERDF) under project MTM2014-60127-P. 


\section{REFERENCES}

1. D. Archambault, T. Munzner, D. Auber: TopoLayout: Multilevel graph layout by topological features. IEEE Trans. Vis. Comput. Graph., 13 (2) (2007), 305-317.

2. D. Archambault, T. Munzner, D. Auber: Grouseflocks: Steerable exploration of graph hierarchy space. IEEE Trans. Vis. Comput. Graph., 14 (4) (2008), 900-913.

3. C. Balbuena, X. Marcote: The k-restricted edge-connectivity of a product of graphs. Discrete Appl. Math., 161 (1-2) (2013), 52-59.

4. B. BollobÁs: Modern Graph Theory (Graduate Texts in Mathematics). Springer, New York, 1998.

5. B. BREŠAR, S. ŠPACAPAN: Edge-connectivity of strong products of graphs. Discuss. Math. Graph Theory, 27 (23) (2007), 333-343.

6. J. Cáceres, C. Hernando, M. Mora, I. M. Pelayo, M. L. Puertas: On the geodetic and the hull numbers in strong product graphs. Comput. Math. Appl., 60 (2010), 3020-3031.

7. G. Chartrand, L. Lesniak: Graphs and Digraphs. Chapman and Hall/CRC, 2005.

8. S. Gavrilets, J. Gravner: Percolation on the fitness hypercube and the evolution of reproductive isolation. J. Theoret. Biol., 184 (1997), 51-64.

9. I. GonzÁlez Yero, J. A. Rodríguez-VelázQuez: Roman domination in cartesian product graphs and strong product graphs. Appl. Anal. Discrete Math., 7 (2) (2013), $262-274$.

10. W. Grüener, R. Giegerich, D. Strothmann, C. Reidys, J. Weber, I. L. HoFACKer, P. F. StAdler, P. Schuster: Analysis of RNA sequence structure maps by exhaustive enumeration. I. neutral networks. Monatsh. Chem., 127 (1996), 355-374.

11. R. Hаммаск: On direct product cancellation of graphs. Discrete Math., 309 (8) (2009), 2538-2543.

12. R. Hammack, W. Imrich, S. KlavŽAr: Handbook of Product Graphs. Second Edition (Discrete Mathematics and Its Applications). Chapman and Hall/CRC, 2011.

13. P. Hell, Z. PAN, T. WONG, X. Zhu: Adaptable chromatic number of graph products. Discrete Math., 309 (21) (2009), 6153-6159.

14. A. Hellwig, L. Volkmann: Maximally edge-connected and vertex-connected graphs and digraphs: A survey. Discrete Math., 308 (15) (2008), 3265-3296.

15. A. Kaveh, K. Koohestani: Graph products for configuration processing of space structures. Comput. Struct., 86 (11-12) (2008), 1219-1231.

16. A. KaveH, R. Mirzaie: Minimal cycle basis of graph products for the force method of frame analysis. Commun. Numer. Methods Eng., 24 (8) (2008), 653-669.

17. A. KaVeh, H. RAHAmi: An efficient method for decomposition of regular structures using graph products. Int. J. Numer. Methods Biomed. Eng., 61 (11) (2004), 17971808.

18. T. KoJima: Bandwidth of the strong product of two connected graphs. Discrete Math., 308 (41) (2008), 1282-1295.

19. M. Lu, G. Chen, X. Xu: On super edge-connectivity of product graphs. Appl. Math. Comput., 207 (2) (2009), 300-306. 
20. E. van Nimwegen, J. P. Crutchfield, M. A. Huynen: Neutral evolution of mutational robustness. Proc. Natl. Acad. Sci. USA, 96 (1999), 9716-9720.

21. J. Ou: On optimizing edge connectivity of product graphs. Discrete Math., 311 (6) (2011), 478-492.

22. C. M. Reidys, P. F. Stadler: Neutrality in fitness landscapes. Appl. Math. Comput., 117 (2001), 321-350.

23. G. SABIdussi: Graph multiplication. Math. Z., 72 (1960), 446-457.

24. S. ŠPACAPAN: Connectivity of Strong Products of Graphs. Graphs Combin., 26 (2010), 457-467.

25. G. Wagner, P. F. Stadler: Quasi-independence, homology and the unity of type: A topological theory of characters. J. Theoret. Biol., 220 (2003), 505-527.

Departamento de Matemática Aplicada I,

Universidad de Sevilla, E.T.S. de Arquitectura,

Avda. Reina Mercedes, 2,

41012 Sevilla

Spain

E-mail: rociomc@us.es

Departamento de Matemática Aplicada I,

Universidad de Sevilla, E.T.S. de Ingeniería Agronómica,

Ctra. Utrera, s/n,

41013 Sevilla

Spain

Email: mcera@us.es

Departamento de Matemática Aplicada I,

Universidad de Sevilla, E.T.S. de Arquitectura,

Avda. Reina Mercedes, 2,

41012 Sevilla

Spain

E-mail: pgvazquez@us.es

Departamento de Matemáticas,

Universidad de Cádiz, E.P.S. de Algeciras,

Avda. Ramón Puyol, s/n,

11202 Algeciras

Spain

E-mail: jcarlos.valenzuela@uca.es
(Received December 20, 2014)

(Revised August 11, 2015) 